\title{
Microglia activation in the brain as inflammatory biomarker of Alzheimer's disease neuropathology and clinical dementia
}

\author{
Zhongmin Xiang ${ }^{\mathrm{a}}$, Vahram Haroutunian ${ }^{\mathrm{a}}$, Lap $\mathrm{Ho}^{\mathrm{a}}$, Dushant Purohit ${ }^{\mathrm{b}}$ and Giulio Maria Pasinetti ${ }^{\mathrm{a}, *}$ \\ ${ }^{a}$ Department of Psychiatry, The Mount Sinai School of Medicine, New York, NY, 10029-6574, USA \\ ${ }^{\mathrm{b}}$ Department of Pathology, The Mount Sinai School of Medicine, New York, NY, 10029-6574, USA
}

\begin{abstract}
The role of microglia-mediated inflammation in the progression of Alzheimer's disease (AD) neuropathology remains unclear. In this study, postmortem brain sections from AD and control cases were subjected to Human Leukocyte Antigen (HLA)DR immunohistochemistry to examine microglia activation in the progression of AD assessed by pre-mortem clinical dementia rating (CDR) and postmortem pathological manifestations of neuritic plaque (NP) and neurofibrillary tangle (NT) according to the Consortium to Establish a Registry for Alzheimer's Disease (CERAD). In both gray and white matter of the entorhinal cortex (EC) and HLA-DR immunostaining increased with the progression of CDR or CERAD NP, and to a lesser degree with CERAD NT. Between CDR stages HLA-DR significance was found in moderate (CDR 2) to severe dementia (CDR 5) where as between CERAD NP stages staining increased significantly from NP 0 (no plaque) to NP 1 (sparse plaques), suggesting increased microglia activation begins with amyloid NP deposition. In the hippocampus, a significant increase in microglia immunostaining was found in the pyramidal cell layer of CA1 as early as CDR 1 , and in the upper molecular layer of the dentate gyrus in CDR 0.5 . This increase continues with the progression of CDR and reaches maximum in CDR 5. When assessed by CERAD NP stages however, a significant increase in microglia immunostaining was found only in mid-to-late stages (NP 3) and reduced staining was seen in NP 5. These results suggest that microglia activation increases with the progression of AD, with the increase varying depending on the involved brain region.
\end{abstract}

Keywords: Alzheimer, proteomic, microglia, inflammation, biomarker

\section{Introduction}

In recent years, a large number of epidemiological studies have addressed the possible protective effect of anti-inflammatory drug use with regard to AD [3]. It is still uncertain however, whether inflammatory mechanisms actually cause damage in AD, or are merely present to remove the debris associated with neurodegenerative events. On a molecular level it is apparent that an inflammatory response accompanies the neu-

\footnotetext{
* Corresponding author: Giulio Maria Pasinetti, M.D., Ph.D., Neuroinflammation Research Laboratories, Department of Psychiatry, Box 1230, Mount Sinai Medical Center, One Gustave L. Levy Place, New York, New York 10029, USA. Tel.: +1 212241 5579; Fax: +1 212876 9042; E-mail: giulio.pasinetti@mssm.edu.
}

ropathologic features of $\mathrm{AD}[1,18]$ though clinical features of inflammation are absent. The complement system appears to be active in the AD brain [16], with generation of the lytic membrane attack complex [24], and presumably with the release of anaphylatoxins. Upregulation of cyclooxygenase (COX)-2 in neurons as a function of the clinical progression of the AD dementia [9] suggests that inflammatory lipids may also be involved in the pathogenesis of the disease [17].

As the major cellular mediators of inflammation, microglia have the characteristics of antigen-presenting tissue macrophages, including Human Leukocyte Antigen (HLA)-DR surface markers [11]. HLA-DR expression is found in microglia assuming either a ramified (resting state) or an amoeboid (reactive or activated state) morphology. Microglia may synthesize 
and secrete $\beta$-amyloid (A $\beta$ ) [26], as well as produce cytokines [25], nitric oxide and superoxide free radicals $[4,5]$ that may aggravate or promote neurodegeneration. Activated microglia increase in AD patients and also in elderly non-AD controls [11]. Activated microglia and astrocytes have been found in abundance near neuritic plaques (NP) and neurofibrillary tangles (NT) in definitive AD cases $[11,21,23]$. However, it is unclear how microglia activation evolves in relation to the clinical progression of $\mathrm{AD}$ pathology or cognitive status.

In our previous study, we found that up-regulation of neuronal COX-2 protein appeared very early in the clinical progression of $\mathrm{AD}$ and preceded the onset of the induction of cytokine gene expression $[9,10]$. In this study, we examined microglia activation in brain tissue sections from AD patients at different stages of cognitive impairment and pathological manifestation by HLA-DR immunohistochemistry. We found that increased microglia immunoreactivity appeared accelerated by amyloid plaque deposition and became significant in the mid- to- late stages of CDR. These results are important in understanding the inflammation process during the pathological progression of $\mathrm{AD}$.

\section{Materials and methods}

\subsection{Postmortem brain samples}

Human postmortem brain samples from AD and nonAD cases were obtained from the Alzheimer's Disease Research Center (ADRC) of the Mount Sinai School of Medicine (MSSM). A multi-step approach based on cognitive and functional status during the last six months of life was applied to the assignment of Clinical Dementia Rating (CDR) scores as previously reported [8]. Subjects were divided into groups on the basis of their CDR scores as following: 0, non-demented; 0.5 , questionable dementia; 1 , mild dementia; 2 , moderate dementia; 5 , very severe dementia. Subjects who died of infectious diseases, sepsis, chronic debilitating diseases (e.g. disseminated cancer) were excluded from the analysis. All the cases were comparable for post-mortem interval (PMI) and age at death.

Brain tissue samples were fixed in $4 \%$ paraformaldehyde, embedded in paraffin, and sectioned at $10 \mu \mathrm{m}$. The extent of neurofibrillary tangle (NT) and $\beta$-amyloid neuritic plaque (NP) pathology was assessed in accordance with the Consortium to Establish a Registry for Alzheimer's Disease (CERAD) neuropathologic battery as described previously [14]. The density of NT and NP were rated on a 4-point scale: 0 , absent; 1 , sparse; 3, moderate, 5, severe.

\subsection{Immunohistochemistry}

Paraffin sections were deparaffinized in Histoclear (National Diagnostics, Atlanta, Geogia), hydrated in descending concentrations of ethanol, rinsed in phosphate-buffered saline (PBS) and post-fixed in $4 \%$ paraformaldehyde for $30 \mathrm{~min}$. Slides were then treated in $10 \mathrm{mM}$ citric buffer ( $\mathrm{pH} \mathrm{6.0)}$ ) at $90-95^{\circ} \mathrm{C}$ for $20 \mathrm{~min}$ to increase antigen reactivity. Endogenous peroxidase activity was blocked by incubation in $3 \% \mathrm{H}_{2} \mathrm{O}_{2}$ for 30 min. Unspecific binding was blocked by incubation with $1 \%$ goat serum. Tissue sections were incubated overnight at $4^{\circ} \mathrm{C}$ in a humidified chamber with rabbit anti-human HLA-DR antibody (1:50, Dako, Carpinteria, CA). Sections were incubated with secondary biotinylated antibody (goat anti-rabbit, 1:200) for $60 \mathrm{~min}$ at room temperature. Immunoreactivities were visualized with $\mathrm{ABC}$ reagent and DAB (Vectastain ABC kit, DAB substrate kit; Vector Laboratories, Burlingame, CA). Control experiments by omission of the primary or secondary antibodies yielded no staining. A set of sections adjacent to those used for immunohistochemistry were stained with hematoxylin to help identify the general structure.

\subsection{Quantifications}

Images of the brain areas of interest were acquired using a high-resolution charge-coupled-device (CCD) camera (Sony, Japan) and analyzed using Bioquant software (Biometrics, Inc., Nashville, TN). Since few microglia stained showing intact cell morphology, making counting the number of cells difficult, we chose to quantify the areas covered by HLA-DR immunostaining as an index of microglia activation. Under constant illumination, threshold value was set so immunopositive cells were identified and the total covered areas in two sampled areas $\left(0.105 \mathrm{~mm}^{2}\right.$ each $)$ in each region were quantified. The raw values were the average from two tissue sections for each case, examined by two independent examiners. To minimize the effect of variations between individual cases, raw data of microglia immunostained area was transformed to a semiquantitive scale similar to that previously reported [9]. HLADR staining in the five cases with the heaviest staining (highest percentage of area covered in area of interest) was averaged as the maximum staining. All cases were then graded on a 0 to 4 scale: $0,<2 \%$ of maximum staining; 1, 2-10\%; 2, 10-30\%; 3, 30-70\%; 4, >70\%. 


\subsection{Statistical analysis}

All data expressed as mean \pm SEM. One-way Analysis of variations (ANOVA) was used to analyze differences among means using Prism program (GraphPad Software, San Diego, CA). Newman-Keuls post-test was used for significance test between pairs. Spearman's correlation analysis was performed to assess the correlation between two parameters using SPSS program (SPSS Inc., Chicago, IL).

\section{Results}

\subsection{Cases}

Specific case information can be found in Table 1 . There was no significant difference in mean age or postmortem interval among the CDR groups (ANOVA, $p>0.05)$.

\subsection{Microglia immunostaining in the entorhinal cortex (EC BM 36-38)}

Brain tissue sections used for immunohistochemistry encompassed EC and dorsal hippocampal formation as shown schematically in Fig. 1. Numbered areas were quantified as stated (see Methods).

HLA-DR immunostained microglia in the EC were found throughout the brain tissue sections, however in general, the density was higher in white matter than in gray matter. Qualitatively in earlier stages of CDR (0.5-1), more microglia assume "resting state" morphology, with elongated secondary and tertiary branches, and processes that are thicker proximal to the cell body and thinner in the more distal branches. In later stages of CDR (2 and 5), more microglia assume "activated state" morphology resembling macrophages, with enlarged cell bodies and short and thickened processes (not shown).

In the EC, a progressive increase in HLA-DR immunostaining was found in both white matter and gray matter, with maximum staining seen in CDR 5, shown in Figs 2(A) and 2(B). HLA-DR in EC immunostaining in the white matter was more pronounced than in gray matter in CDR 0, and then became comparable from CDR 1 to 5 . In the gray matter, (GM), prominent increase in HLA-DR immunostaining was found only in moderate $(\mathrm{CDR} 2, p<0.05)$ and severe dementia (CDR5, $p<0.001)$ groups when compared with non-demented controls (CDR 0). In the white matter,

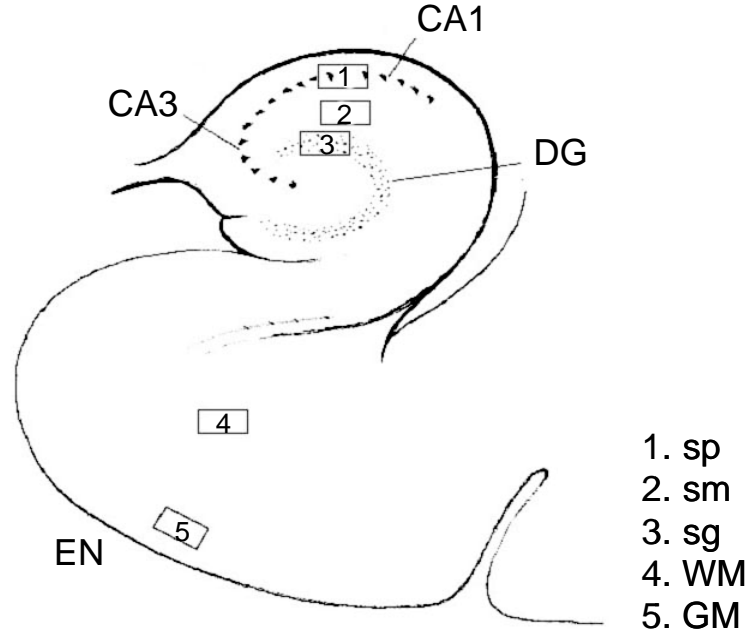

Fig. 1. Schematic drawing of hippocampal formation. Brain sections of dorsal hippocampal formation were immunostained for human HLA-DR. Numbered areas indicate the areas for quantification. Abbreviations: CA3, CA1, pyramidal layers; DG: dentate gyrus; sp: stratum pyramidale; sm: stratum moleculare; sg: stratum granulosum of the hippocampus; EN: entorhinal cortex; GM: gray matter; WM: white matter.

(WM), significant increase was found only in severe dementia group (CDR5, $p<0.01)$.

Next, samples were examined to determine whether or not HLA-DR immunostaining correlated with neuropathologic markers such as neuritic plaques (NP) and neurofibrillary tangles (NT). All cases were regrouped according to their CERAD scores of NP (Fig. 2C) or NT (Fig. 2D) in the EC. In the gray matter, there was very little HLA-DR staining in cases with no plaque (NP 0). A significant increase in HLA-DR staining was found in NP $1(p<0.05)$ cases with sparse plaque pathology. Staining was comparable from sparse (NP 1) to moderate (NP3) plaque pathology, and increased again in NP 5 with abundant plaques. There was no significant difference between NP 1, 3 and 5. In the white matter, there was more pronounced HLA-DR staining at NP 0 when compared with gray matter staining, however there was not significant change in HLA-DR staining with the progression of plaque pathology.

When assessed by CERAD NT stages (Fig. 2D), a trend of increased staining was found in both gray matter and white matter, but was not statistically significant.

\subsection{Microglia immunostaining in the hippocampus}

In the hippocampus, HLA-DR immunostaining was quantified in three sub-regions: pyramidal cell layer of 
Table 1

\begin{tabular}{|c|c|c|c|c|}
\hline \\
\hline CDR & Postmortem & $\begin{array}{c}\text { Sex } \\
\text { interval (min) }\end{array}$ & $\begin{array}{c}\text { Age } \\
\text { of death }\end{array}$ & Cause of death \\
\hline 0 & 181 & $\mathrm{f}$ & 79 & Cardio respiratory failure \\
\hline 0 & 310 & $\mathrm{f}$ & 91 & Cardio respiratory arrest \\
\hline 0 & 340 & $\mathrm{f}$ & 82 & Cardio pulmonary arrest \\
\hline 0 & 205 & $\mathrm{f}$ & 82 & n.a. \\
\hline 0 & 230 & $\mathrm{f}$ & 82 & Pneumonia, Bronchopneumonia, cerebral emblous \\
\hline 0.5 & 315 & $\mathrm{f}$ & 94 & Cardiac failure \\
\hline 0.5 & 240 & $\mathrm{f}$ & 87 & Myocardial infarction \\
\hline 0.5 & 370 & $\mathrm{f}$ & 73 & Myocardial infarction \\
\hline 0.5 & 255 & $\mathrm{~m}$ & 69 & Cancer of lung \\
\hline 0.5 & 300 & $\mathrm{f}$ & 89 & Cardiorespiratory arrest, acute myocardial infarction \\
\hline 0.5 & 147 & $\mathrm{f}$ & 90 & Acute myocardial infarction \\
\hline 1 & 185 & $\mathrm{~m}$ & 98 & n.a. \\
\hline 1 & 160 & $\mathrm{f}$ & 99 & Cardio respiratory failure \\
\hline 1 & 155 & $\mathrm{f}$ & 90 & Cardio pulmonary arrest \\
\hline 1 & 240 & $\mathrm{f}$ & 83 & Myocardial infarction \\
\hline 1 & 580 & $\mathrm{f}$ & 103 & Myocardial infarction \\
\hline 1 & 340 & $\mathrm{~m}$ & 80 & Myocardial infarction, a arteriosclerotic heart disease \\
\hline 1 & 175 & $\mathrm{f}$ & 84 & Cardio-respiratory arrest \\
\hline 2 & 185 & $\mathrm{f}$ & 90 & n.a. \\
\hline 2 & 120 & $\mathrm{~m}$ & 92 & Cardio respiratory failure \\
\hline 2 & 160 & $\mathrm{f}$ & 93 & Cardio pulmonary arrest \\
\hline 2 & 255 & $\mathrm{f}$ & 97 & Cardio respriatory arrest \\
\hline 2 & 375 & $\mathrm{~m}$ & 74 & Cardiopulmonary arrest \\
\hline 2 & 185 & $\mathrm{f}$ & 94 & Mycardial infarction \\
\hline 2 & 210 & $\mathrm{f}$ & 86 & Acute mycardial infarction \\
\hline 5 & 285 & $\mathrm{f}$ & 93 & Cardio respiratory failure \\
\hline 5 & 250 & $\mathrm{f}$ & 86 & Acute myocardial infarct \\
\hline 5 & 198 & $\mathrm{f}$ & 94 & Acute mycardial infarction \\
\hline 5 & 235 & $\mathrm{f}$ & 102 & Cardio pulmonary arrest \\
\hline 5 & 375 & $\mathrm{~m}$ & 74 & n.a. \\
\hline 5 & 260 & $\mathrm{~m}$ & 94 & n.a. \\
\hline
\end{tabular}

CA1 (sp), granule cell layer of dentate gyrus (sg), and the upper molecular layer (sm), shown in Fig. 3 (see scheme Fig. 1). There was minimal staining in all three sub-regions in CDR 0. HLA-DR immunostaining increased progressively from CDR 0.5 to CDR 5 (Figs 3A and $3 \mathrm{~B})$. In CA1 (sp), significant increases were found in mild (CDR 1) to severe dementia (CDR 5) cases compared to non-demented controls (CDR 0). In the upper molecular layer (sm), significant increases were found in questionable (CDR 0.5) to severe dementia (CDR 5). In granule cell layer of dentate gyrus (sg), HLA-DR immunostaining was less then that found in the CA1 or molecular layers at each CDR (0.5 to 5), however significant increases were found in moderate (CDR 2) to severe dementia (CDR 5).

With regard to CERAD pathological staging by neuritic plaques (NP), it was found that from no plaque (NP 0) to moderate plaque (NP 3) stages, there was an increase in HLA-DR immunostaining in all three sub-areas quantified (shown in Fig. 3C), with the granular cell layer having the least staining. HLA-DR im-

munostaining in moderate plaque (NP 3) stage is significantly higher in all three sub-areas compared to no plaque (NP 0) stage, however, at the severe plaque stage NP 5, staining decreased to levels seen in NP 0 or NP1.

CERAD pathological staging by neurofibrillary tangle (NT) (Fig. 3D) revealed an increase in HLA-DR staining, with a significant increase found only in the severe NT pathological stage (NT 5) when compared to that of stages with absent or sparse NT pathology (NT 0-1).

\subsection{Correlation of microglia immunostaining with clinical and pathological manifestations}

The aforementioned study examined HLA-DR microglia immunostaining in relation to cases grouped by either clinical dementia stages (CDR), or by pathological stages (CERAD) using neuritic plaques(NP) and neurofibrillary tangles (NT). However, a more integrated analysis is needed to determine whether there is a correlation between HLA-DR immunostaining and 
A
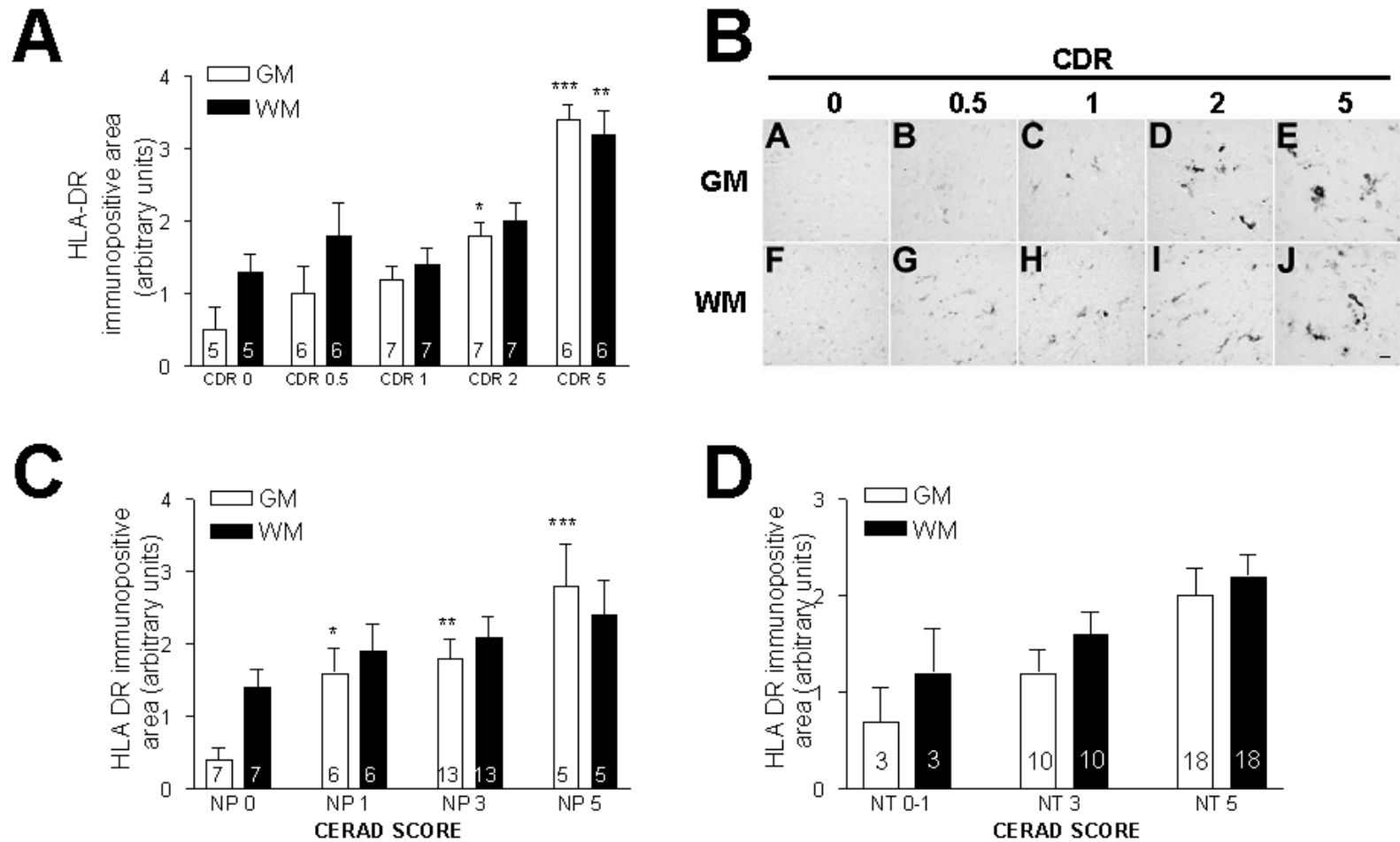

Fig. 2. Microglia immunostaining in the entorhinol cortex of cases at progressive AD stages. HLA-DR immunostaining was quantified and scored semi-quantitatively in white matter (WM) and gray matter (GM) of the entorhinol cortex (see Methods). Panel A. Summary of HLA-DR immunostaining in the progression of AD by CDR. Panel B. Typical staining of microglia in gray matter (A-E) and white matter (F-J) of the entorhinal cortex from CDR 0 to CDR 5. Scale bar, $5 \mu \mathrm{m}$. Panel C. Summary of HLA-DR immunostaining in the progression of AD by CERAD score of neuritic plaques (NP). Panel D. Summary of HLA-DR immunostaining in the progression of AD by CERAD score of neuritic plaques (NT). NT 0 and NT 1 are combined due to insufficient cases. All data expressed as mean \pm SEM, number within bar indicates number of cases. Asterisks indicate significance compared to CDR 0 (ANOVA, $p<0.05$; Neuman-Keul post test, *, $p<0.05$; **, $p<0.01$; ***, $p<0.001$ ).

all the pathological indexes in each case. For this purpose, Spearman's correlation coefficient ( $\rho$ was calculated between any two indexes, listed in Table 2.

CDR correlates with CERAD of both NP and NT ( $\rho$ range from 0.481 to $0.825, p<0.01$ ) with the exception of CERAD NT in the EC $(\rho=0.277, p>0.05)$, shown in the first column, confirming overall the validity of CDR in relation to pathology. While CERAD NP in the EC (BM 36,38) correlates with HLA-DR immunostaining in the gray matter $(\rho=0.546, p<0.01)$ but not in the white matter $(\rho=0.26, p>0.05)$, CERAD NT in the entorhinol cortex correlates with HLADR immunostaining in the white matter $(\rho=0.363$, $p<0.05)$ but not in the gray matter $(\rho=0.344$, $p>0.05$ ), shown in the second and third column, Table 2 .

In the hippocampus, CERAD NP correlates with HLA-DR immunostaining in all three sub-regions ( $\rho$ range from 0.452 to $0.489, p<0.05$ for molecular layer, $p<0.01$ for CA1 and granular cell layer). CERAD NT correlates with HLA-DR immunostaining only in CA1 $(\rho=0.462, p<0.05)$, but not in the granular cell layer $(\rho=0.263, p>0.05)$ or molecular layer $(\rho=0.345, p>0.05)$.

\section{Discussion}

This study suggests that there is increased microglia HLA-DR immunostaining with the progression of AD CDR stages, with the most prominent staining occurring in the gray matter of the EC, as well as in the pyramidal cell layer of the CA1 region of the hippocampus. Dramatic changes were also seen in the molecular layer of the hippocampus, while increases in the dentate gyrus (DG) were modest. Microglia activation in the gray matter of the EC appears to begin with the onset of AD NP pathology.

Activated microglia represent the cellular component of the inflammatory response in the AD brain [13], and are capable of antigen processing and presenting. Activated microglia may also secrete neurotoxins that 


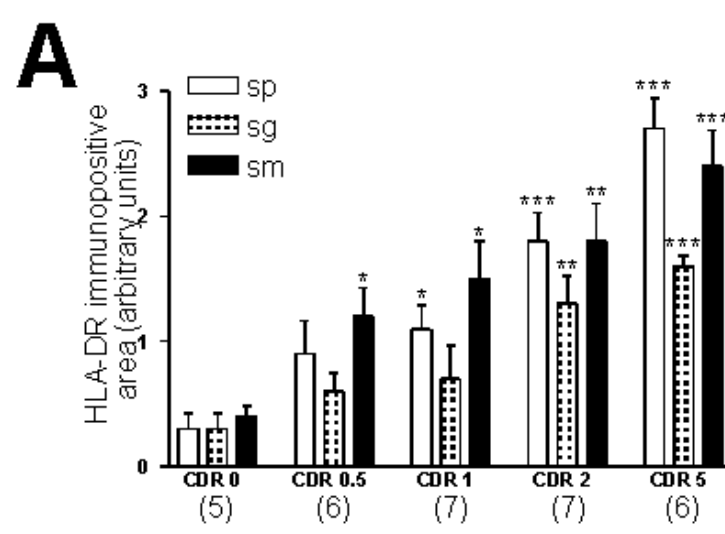

B
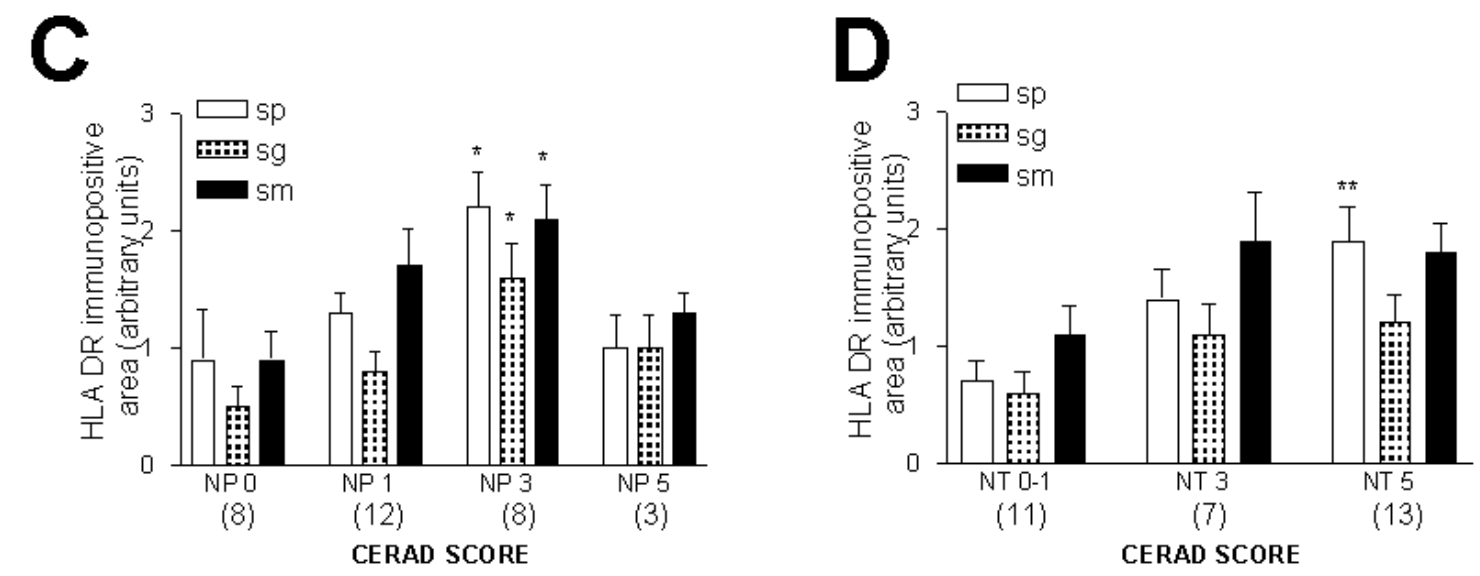

Fig. 3. Microglia immunostaining in the hippocampal formation of cases at progressive AD stages. HLA-DR immunostaining was quantified and scored semi-quantitatively in three sub-regions in the hippocampus: pyramical cell layer of CA1 (sp), granule cell layer of dentate gyrus (sg), and upper molecular layer (sm) (see Methods). Panel A. Summary of HLA-DR immunostaining in the progression of AD by CDR. Panel B. Typical staining of microglia in pyramical cell layer of CA1 (sp, a-e), granule cell layer of dentate gyrus (sg, f-j) and upper molecular layer (sm, k-o) of hippocampus from CDR 0 to CDR 5. Panel C. Summary of HLA-DR immunostaining in the progression of AD by CERAD scores of neuritic plaque (NP). Panel D. Summary of HLA-DR immunostaining in the progression of AD by CERAD scores of neuritic plaque (NT). NT 0 and NT 1 are combined due to insufficient cases in NT 0. All data expressed as mean \pm SEM, number in bracket indicates number of cases. Asterisks indicate significance compared to CDR 0 (ANOVA, $p<0.05$; Neuman-Keul post test, ${ }^{*}, p<0.05 ; * *, \mathrm{p}<0.01 ; * * *, p<0.001$ ).

contribute to neuronal loss in $\mathrm{AD}[6,7]$. It is unclear whether these processes represent a reaction to neurodegeneration in $\mathrm{AD}$, however, it is likely that they contribute to neuronal loss. HLA-DR immunopositive microglia also express leukocyte common antigen (LCA) and FcgRI or FcgRII receptors that promote phagocytosis [12]. Such reactive microglial cells are often found within or intimately associated with senile plaques [22] where they express complement receptors CR3 and CR4. These receptors bind to $\mathrm{iC} 3 \mathrm{~b}$, a potent opsonin (a cell-associated tag for phagocytosis) on target cell surfaces [19]. Thus microglia may play a role in complement-mediated phagocytosis in the brain. The local degenerative environment of the AD brain, which contains an abundance of $\beta$-amyloid $(\mathrm{A} \beta)$, neurofibrillary tangles, dystrophic neurites, neuropil threads, and possibly injured neurons ,may be favorable for complement system activation.

Cultured microglia can secrete $\mathrm{A} \beta$ and metabolize amyloid precursor protein (APP) in a manner that might favor $A \beta$ deposition [2]. Thus microglia may be involved in the conversion of non-fibrillar $\mathrm{A} \beta$ into amyloid fibrils, a role similar to that ascribed to peripheral macrophages in systemic amyloidosis [15]. Activated microglia are also capable of producing a variety of pro-inflammatory mediators [20] and potentially neurotoxic substances [27] that could contribute to localized, or more widespread, brain injury. These include complement cytokines, reactive oxygen intermediates, and nitric oxide, among others [4,5]. Moreover, conditions in the $\mathrm{AD}$ brain may enhance production of inflammation-related molecules. $\mathrm{A} \beta$, for example, is a 
Table 2

Spearman $\rho$ coefficient table

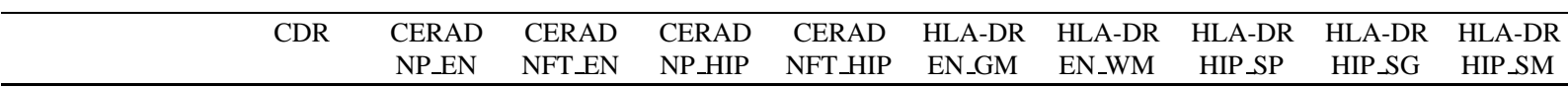

CDR

CERAD NP EN $\quad 0.584(* *) \quad 1$

$\begin{array}{llll}\text { CERAD NFT EN } & 0.277 & 0.401(*) & 1\end{array}$

$\begin{array}{llll}\text { CERAD NP HIP } & 0.493(* *) & 0.773(* *) & 0.29\end{array}$

$\begin{array}{llll}\text { CERAD NFT HIP } & 0.481(* *) & 0.613(* *) & 0.590(* *)\end{array}$

HLA-DR EN GM $0.744(* *) \quad 0.546(* *) \quad 0.3$

$\begin{array}{llll}\text { HLA-DR EN WM } & 0.551(* *) & 0.26 & 0.363(*)\end{array}$

HLA-DR HIP SP $\quad 0.825(* *) \quad 0.451\left(^{*}\right) \quad 0.27$

$\begin{array}{llll}\text { HLA-DR HIP SG } & 0.692(* *) & 0.375(*) & 0.197\end{array}$

$\begin{array}{llll}\text { HLA-DR HIP SM } & 0.741(* *) & 0.362 & 0.302\end{array}$

$\begin{array}{cc}1 & \\ 0.543(* *) & 1\end{array}$

$0.579(* *) \quad 0.465(* *)$

$0.301 \quad 0.316$

$0.486(* *) \quad 0.462(*)$

$0.489(* *) \quad 0.263$

$0.452(*) \quad 0.345$

1

$0.819(* *) \quad 1$

$0.808(* *) \quad 0.724(* *)$

$\begin{array}{llll}0.669(* *) & 0.607(* *) & 0.866(* *) & 1\end{array}$

$\begin{array}{llll}0.780(* *) & 0.791(* *) & 0.842(* *) & 0.789(* *)\end{array}$

1

*Correlation is significant at the 0.05 level (2-tailed).

**Correlation is significant at the 0.01 level (2-tailed).

Abbreviations: CERAD: Consortium to Establish a Registry for Alzheimer's Disease; CDR: Clinical Dementia Rating; NP: Neuritic Plaque; NFT: Neurofibrillary Tangle; HIP: Hippocampus; GM: Gray Matter; WM: White Matter; DG: dentate gyrus; EC: Entorhinal Cortex; SP: Stratum Pyramidale; SM: stratum moleculare; SG: stratum granulosum.

particularly potent stimulator of the microglial production of IL- 6 , IL-1, TNF- $\alpha$, nitric oxide, and superoxide free radicals $[4,5]$.

It was hypothesized in this study that microglia mediated responses at later stages of the clinical progression of $\mathrm{AD}$ dementia may play an important role in fueling a cycle of inflammatory cascades leading to accelerated AD neuropathology. Thus the precise identification of the CDR stages at which pro-inflammatory molecules may become available for microglia activation will facilitate the development of anti-inflammatory strategies aimed at slowing the clinical progression of AD. These data suggest that in respect to CDR score, microglia activation in the hippocampal formation (molecular layer) temporally follows the induction of the proinflammatory COX-2 protein content in the hippocampal pyramidal neuron layer (CA1-CA3) and overlaps the peak of median neurofibrillary tangles (NFT) CERAD ratings [8]. Ongoing studies in the lab are further exploring the relationship between microglia activation and amyloid plaque maturation (diffuse vs neuritic plaques) at different stages of disease.

\section{Acknowledgements}

Supported by NIA AG14766 and AG02219 (subproject II) to GMP. We thank Ms. Isabela Diaconescu and Dr. Breton Stetka for editorial revision of the manuscript.

\section{References}

[1] P.S. Aisen, Inflammation and Alzheimer disease, Mol Chem Neuropathol 28 (1996), 83-88.
[2] J. Bauer, G. Konig, S. Strauss et al., In-vitro matured human macrophages express Alzheimer's beta A4-amyloid precursor protein indicating synthesis in microglial cells, FEBS Lett $\mathbf{2 8 2}$ (1991), 335-340.

[3] J.C. Breitner, Epidemiologic clues to the causes and routes to prevention of Alzheimer disease, J Neural Transm Suppl 59 (2000), 251-254.

[4] C.A. Colton and D.L. Gilbert, Production of superoxide anions by a CNS macrophage, the microglia, FEBS Lett 223 (1987), 284-288.

[5] C.W. Cotman, The beta-amyloid peptide, peptide selfassembly, and the emergence of biological activities. A new principle in peptide function and the induction of neuropathology, Ann N Y Acad Sci 814 (1997), 1-16.

[6] D.A. Dzenko, R.B. Weltzien and J.S. Pachter, Suppression of A beta-induced monocyte neurotoxicity by antiinflammatory compounds, J Neuroimmunol 80 (1997), 6-12.

[7] D. Giulian, J. Li, B. Leara and C. Keenen, Phagocytic microglia release cytokines and cytotoxins that regulate the survival of astrocytes and neurons in culture, Neurochem Int $\mathbf{2 5}$ (1994), 227-233.

[8] V. Haroutunian, D.P. Purohit, D.P. Perl et al., Neurofibrillary tangles in nondemented elderly subjects and mild Alzheimer disease, Arch Neurol 56 (1999), 713-718.

[9] L. Ho, D. Purohit, V. Haroutunian et al., Neuronal cyclooxygenase 2 expression in the hippocampal formation as a function of the clinical progression of Alzheimer disease, Arch Neurol 58 (2001), 487-492.

[10] J.D. Luterman, V. Haroutunian, S. Yemul et al., Cytokine gene expression as a function of the clinical progression of Alzheimer disease dementia, Arch. Neurol. 57 (2000), 11531160.

[11] L.A. Mattiace, P. Davies and D.W. Dickson, Detection of HLA-DR on microglia in the human brain is a function of both clinical and technical factors, Am J Pathol 136 (1990), 1101-1114.

[12] P.L. McGeer, H. Akiyama, S. Itagaki and E.G. McGeer, Immune system response in Alzheimer's disease, Can J Neurol Sci 16 (1989), 516-527.

[13] P.L. McGeer, S. Itagaki, H. Tago and E.G. McGeer, Reactive microglia in patients with senile dementia of the Alzheimer type are positive for the histocompatibility glycoprotein HLADR, Neurosci Lett 79 (1987), 195-200. 
[14] S.S. Mirra, Neuropathological assessment of Alzheimer's disease: the experience of the Consortium to Establish a Registry for Alzheimer's Disease, Int Psychogeriatr 9(1) (1997), 263-268.

[15] K, Miura, S. Baba, H. Shirasawa, S.T. Ju, A.S. Cohen and T. Shirahama, Intraperitoneal amyloid formation by amyloid enhancing factor-rich macrophages in ascitic fluid, Virchows Arch B Cell Pathol Incl Mol Pathol 62 (1992), 245-250.

[16] G.M. Pasinetti, Inflammatory mechanisms in neurodegeneration and Alzheimer's disease: the role of the complement system, Neurobiol Aging 17 (1996), 707-716.

[17] G.M. Pasinetti, Cyclooxygenase and inflammation in Alzheimer's disease: experimental approaches and clinical interventions, J Neurosci Res 54 (1998), 1-6.

[18] G.M. Pasinetti and P.S. Aisen, Cyclooxygenase-2 expression is increased in frontal cortex of Alzheimer's disease brain, Neuroscience 87 (1998), 319-324.

[19] J.M. Rozemuller, V. van d and P. Eikelenboom, Activated microglia and cerebral amyloid deposits in Alzheimer's disease, Res Immunol 143 (1992), 646-649.

[20] M. Sawada, N. Kondo, A. Suzumura and T. Marunouchi, Production of tumor necrosis factor-alpha by microglia and astrocytes in culture, Brain Res 491 (1989), 394-397.

[21] L.G. Sheffield, J.G. Marquis and N.E. Berman, Regional distribution of cortical microglia parallels that of neurofibrillary tangles in Alzheimer's disease, Neurosci Lett 285 (2000), 165168.

22] S.D. Styren, W.H. Civin and J. Rogers, Molecular, cellular, and pathologic characterization of HLA-DR immunoreactivity in normal elderly and Alzheimer's disease brain, Exp Neurol 110 (1990), 93-104.

[23] D.R. Thal, T. Arendt, G. Waldmann et al., Progression of neurofibrillary changes and PHF-tau in end-stage Alzheimer's disease is different from plaque and cortical microglial pathology, Neurobiol Aging 19 (1998), 517-552.

[24] S. Webster, L.F. Lue, L. Brachova et al., Molecular and cellular characterization of the membrane attack complex, C5b-9, in Alzheimer's disease, Neurobiol Aging 18 (1997), 415-421.

[25] S.L. Wesselingh, K. Takahashi, J.D. Glass, J.C. McArthur, J.W. Griffin and D.E. Griffin, Cellular localization of tumor necrosis factor mRNA in neurological tissue from HIV-infected patients by combined reverse transcriptase/polymerase chain reaction in situ hybridization and immunohistochemistry, J Neuroimmunol 74 (1997), 1-8.

[26] H.M. Wisniewski and J. Wegiel, The role of microglia in amyloid fibril formation, Neuropathol Appl Neurobiol 20 (1994), 192-194.

[27] J. Yao, J.E. Keri, R.E. Taffs and C.A. Colton, Characterization of interleukin-1 production by microglia in culture, Brain Res 591 (1992), 88-93. 


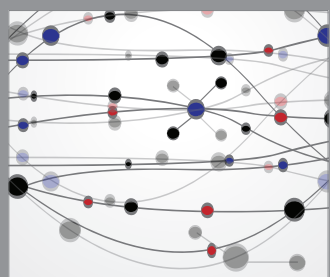

The Scientific World Journal
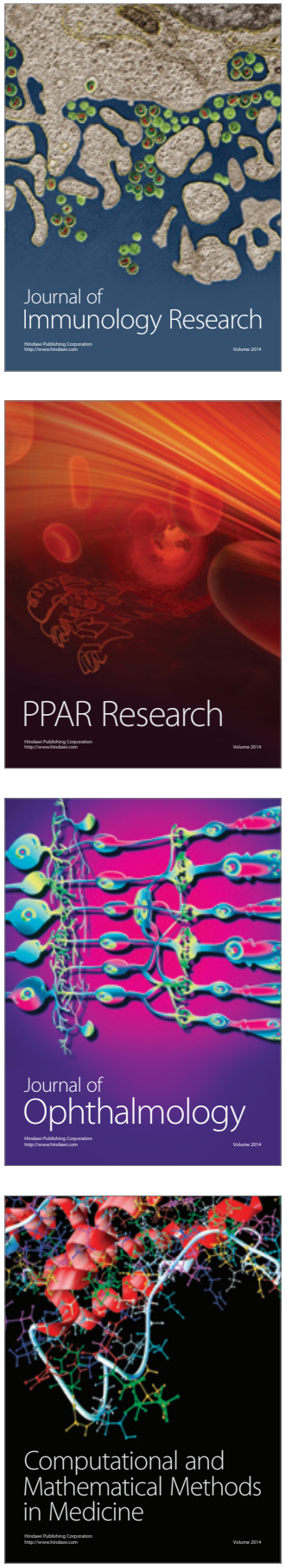

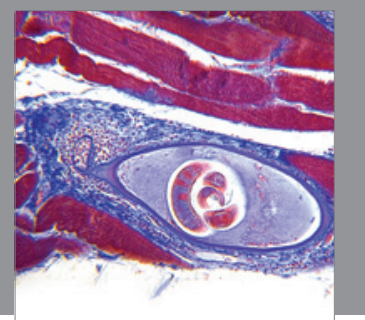

Gastroenterology

Research and Practice
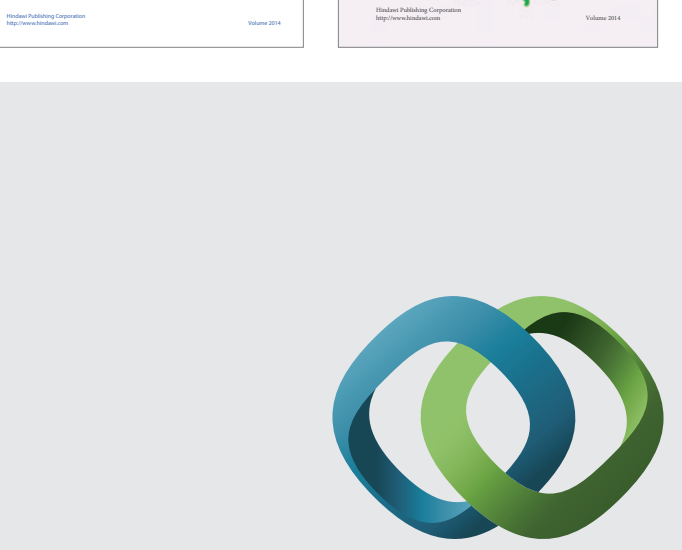

\section{Hindawi}

Submit your manuscripts at

http://www.hindawi.com
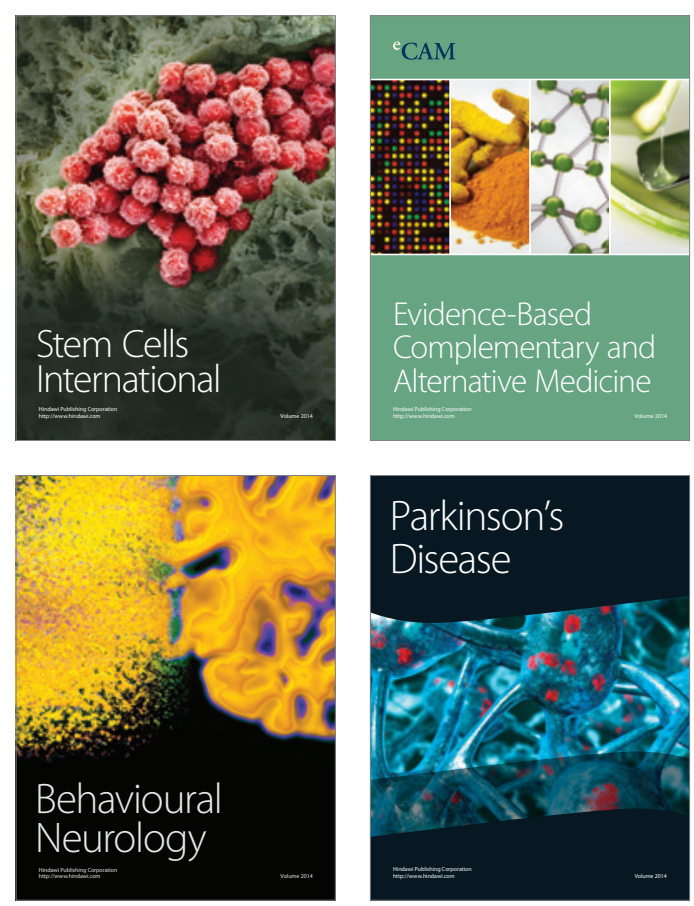

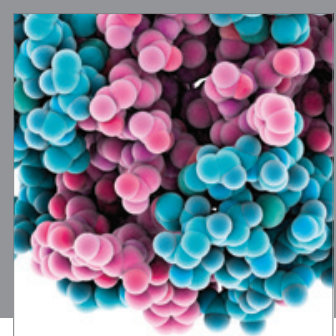

Journal of
Diabetes Research

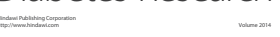

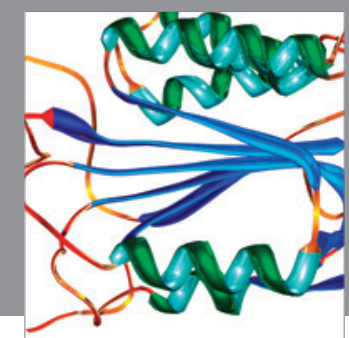

Disease Markers
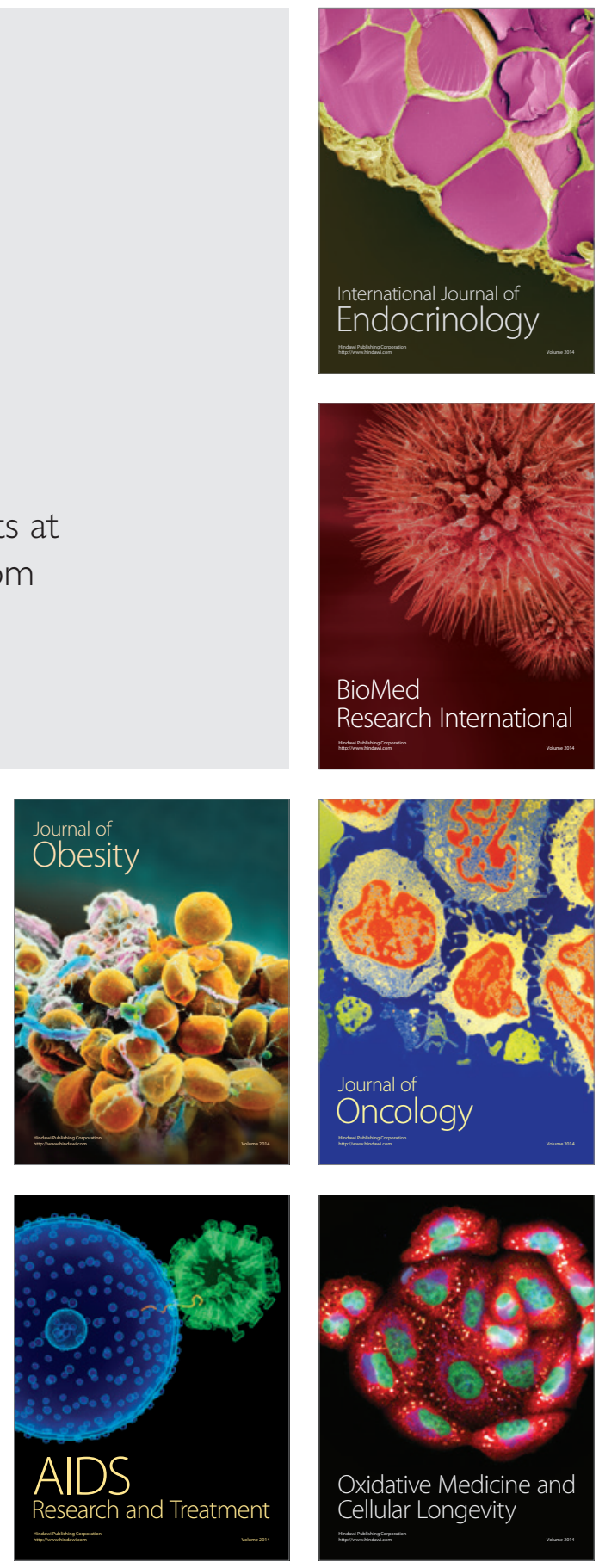